INPLASY

PROTOCOL

To cite: Tan et al. Prognostic and clinicopathological value of PD-L1 expression in patients with esophageal cancer: a systematic review and meta-analysis. Inplasy protocol 202160051. doi: 10.37766/inplasy2021.6.0051

Received: 15 June 2021

Published: 16 June 2021

Corresponding author: Hui Tan

tanhuiwy@126.com

Author Affiliation:

Chongqing Three Gorges

Medical College.

Support: Chongqing Research Project.

Review Stage at time of this submission: Preliminary searches.

Conflicts of interest: None declared.

\section{Prognostic and clinicopathological value of PD-L1 expression in patients with esophageal cancer: a systematic review and meta-analysis}

\author{
Tan, $\mathrm{H}^{1}$; Zhu, Y2; Wen, S3; Deng, $\mathrm{X}^{4}$.
}

Review question / Objective: The purpose of this systematic review and meta-analysis is to study the expression of immune checkpoints PD-L1 in esophageal cancer, and whether it affects the survival of patients with esophageal cancer and clinicpathological features.

Condition being studied: Esophageal cancer is a common and often fatal cancer that it is the sixth leading cause of cancerrelated deaths worldwide, although the advances in multidisciplinary therapeutic strategies have improved the prognosis of esophageal cancer, overall outcome is still dismal and novel approaches of treatment are needed.Recent clinical studies have shown promising clinical activity of programmed cell death ligand 1(PD-L1) targeting antibodies in certain malignancies, including esophageal cancer. Researches also demonstrated that PD-L1 protein expression on cancer cells may predict favorable response to anti-PD-L1 therapy. PD-L1 expression in patients with esophageal cancer remains controversial. Some investigations showed that overexpression of PD-L1 forecasted poor survival in patients with esophageal cancer, but other investigations presented negative results. To investigate this question, we conducted a meta-analysis.

INPLASY registration number: This protocol was registered with the International Platform of Registered Systematic Review and Meta-Analysis Protocols (INPLASY) on 16 June 2021 and was last updated on 16 June 2021 (registration number INPLASY202160051).

\section{INTRODUCTION}

Review question / Objective: The purpose of this systematic review and meta-analysis is to study the expression of immune checkpoints PD-L1 in esophageal cancer, and whether it affects the survival of patients with esophageal cancer and clinicpathological features.

Condition being studied: Esophageal cancer is a common and often fatal cancer 
that it is the sixth leading cause of cancerrelated deaths worldwide, although the advances in multidisciplinary therapeutic strategies have improved the prognosis of esophageal cancer, overall outcome is still dismal and novel approaches of treatment are needed. Recent clinical studies have shown promising clinical activity of programmed cell death ligand 1(PD-L1) targeting antibodies in certain malignancies, including esophageal cancer. Researches also demonstrated that PD-L1 protein expression on cancer cells may predict favorable response to anti-PD-L1 therapy. PD-L1 expression in patients with esophageal cancer remains controversial. Some investigations showed that overexpression of PD-L1 forecasted poor survival in patients with esophageal cancer, but other investigations presented negative results. To investigate this question, we conducted a meta-analysis.

\section{METHODS}

Search strategy: (esophageal neoplasms OR esophagus neoplasm OR cancer of esophagus OR esophagus cancer OR esophageal cancer OR esophageal squamous cell carcinoma) AND (programmed cell death ligand 1 OR PD-L1 OA B7-H1).

Participant or population: Patients with esophageal cancer confirmed by pathological examination.

Intervention: The exposure group is patients with esophageal cancer in high expression of PD-L1.

Comparator: The exposure group is patients with esophageal cancer in low expression of PD-L1.

Study designs to be included: Study designs to be included: randomized controlled trials, case-control study and cohort studies. Only consider English studies and published as full-text articles.

Eligibility criteria: Inclusion criteria: (1)the object of study is esophageal cancer confirmed by pathological examination; (2)the statistics record of at least one of the following indicators: prognostic indicators of OS, DFS or RFS; (3) literature published in English. Exclusion criteria: (1) if the objective of the study was non-esophageal cancer; (2) Non-comparative study; (3)complete clinical data was not provided, contact first author and no response was received; (4) repeated literature.

Information sources: We comprehensively searched the following databases for relevant studies up to May 30, 2021: PubMed, Embase, the Cochrane library and Web of Science. The following information was extracted from these studies: first author's name, year of publication, country of the patients, research type, number of patients, patients age, cut-off of PD-L1, prognostic indicators and clinicopathological features.

Main outcome(s): Over survival (OS), disease free survival (DFS) and recurrence free survival (RFS).

Quality assessment / Risk of bias analysis: According to the searching strategies, two authors independently searched relevant researches and screened literature by their titles and abstracts. After the initial screening, these articles satisfied the inclusion criteria evaluated with full texts for final inclusion. In this process, the third author would resolve the discrepancy. Two authors collected data form involved literature and compared outcome data independently. The quality of studies would be evaluated by the Newcastle-Ottawa Scale (NOS). Three factors were used for evaluation: (1) patient selection; (2) comparability of research groups; (3) assessment of outcome. This quality assessment scale had a maximum score of 9 , studies with scores $\geq 6$ were considered to be high quality.

Strategy of data synthesis: The hazard ratio (HR) with $95 \% \mathrm{Cl}$ was utilized to assess the relationship between the expression of PDL1 and OS, DFS, RFS. The merged ORs and the $95 \%$ Cls were utilized to quantitatively 
determine the relationship between PD-L1 and clinicopathological feature of the patient. The heterogeneity between included studies was examined using $I^{2}$ and $Q$ text. If $\mathrm{p} 50 \%$, which demonstrated significant heterogeneity, the fixed effect model was used for meta-analysis. If there was significant heterogeneity, the random model was used. In this analysis, p-values $<0.05$ were considered statistically significant.

Subgroup analysis: If the necessary data are available, a subgroup analysis will be performed on the different cut-off values for PD-L1 positive, different ethnicity.

Sensitivity analysis: If necessary, a sensitivity analysis will also be carried out, the purpose of which is to eliminate the influence of trials that may have a high risk of bias on the reliability and robustness of the assessment results. The results of the sensitivity analysis will be reported in the summary table.

Language: English.

Country(ies) involved: China.

Keywords: Esophageal cancer; PD-L1;

Prognosis; Meta-analysis.

Contributions of each author:

Author 1 - Hui Tan.

Email: tanhuiwy@126.com

Author 2 - Yumiao Zhu.

Author 3 - Shiliu Wen.

Author 4 - Xuesong Deng.

Email: dengxssxu@126.com 